\title{
LETTER \\ Joint Channel Estimation and Phase Noise Suppression for OFDM Systems
}

\author{
Yong-Hwa $\mathrm{KIM}^{\dagger}$, Nonmember and Seong-Cheol $\mathrm{KIM}^{\dagger \dagger \mathrm{a})}$, Member
}

SUMMARY Phase noise (PHN) can cause the common phase error (CPE) and the inter-carrier interference (ICI), both of which impair the accurate channel estimation in orthogonal frequency division multiplexing (OFDM) systems. In this letter, we build a new signal model parameterized by the channel impulse response, the CPE and the ICI. Based on this model, we derive the maximum likelihood estimator (MLE) and the minimum mean square error estimator (MMSEE). Simulation results show that the proposed schemes significantly improve the performance of OFDM systems in the presence of PHN.

key words: maximum-likelihood, minimum mean square error, orthogonal frequency-division multiplexing (OFDM), phase noise (PHN)

\section{Introduction}

Orthogonal frequency-division multiplexing (OFDM) is an attractive technique to support high rate data transmission over frequency selective fading channels. However, it is known to be very sensitive to frequency synchronization and imperfect channel estimation [1].

The phase noise (PHN) induced by oscillator instability can cause the common phase error (CPE) and the intercarrier interference (ICI), which may result in significant performance degradation [2], [3]. Under the assumption that the channel estimation is perfect, several PHN mitigation schemes for OFDM systems are reported in [3], [4]. However, the perfect channel estimation assumption may not be valid in the practical systems [1].

In this letter, we propose a new parametric model for OFDM systems in the presence of PHN. Based on this model, we propose two pilot symbol aided decision directed (PADD) approaches. One is a maximum likelihood estimator (MLE) and the other is a minimum mean square error estimator (MMSEE). Along with the estimation techniques, we also examine the detection method and the issue of a pilot sub-carrier placement.

The following notations are used throughout this letter: $\operatorname{diag}\left(\boldsymbol{A}_{0}, \cdots, \boldsymbol{A}_{k-1}\right)$ represents a block diagonal matrix whose diagonal blocks are $\boldsymbol{A}_{0}, \cdots, \boldsymbol{A}_{k-1}$. $[\boldsymbol{A}]_{p, q}$ denotes the $(p, q)$ th element of $\boldsymbol{A} . \quad \boldsymbol{I}_{t}$ stands for a $t$-by- $t$ identity matrix, $[\boldsymbol{a}]_{p}$ is the $(p)$ th entry of vector $\boldsymbol{a}$ and $((\cdot))_{N}$ denotes a modulo- $N$ operation.

Manuscript received January 24, 2008.

Manuscript revised June 1, 2008.

†The author is with the Korea Electrotechnology Research Institute (KERI), Korea.

${ }^{\dagger \dagger}$ The author is with School of Electrical Engineering and Computer Science, Seoul National University, Seoul, Korea.

a) E-mail: sckim@maxwell.snu.ac.kr

DOI: 10.1093/ietcom/e91-b.10.3371

\section{System Model}

We consider an OFDM system with $N$ sub-carriers being composed of a data sub-carrier set $S_{D}$ with $N_{D}$ sub-carriers and a pilot sub-carrier set $S_{P}$ with $N_{P}$ sub-carriers. The time-domain OFDM signal is generated by the IDFT and a cyclic prefix (CP) of length $N_{C P}$ is appended at the beginning of each time-domain OFDM signal. After CP removal and taking the DFT, the $N$-dimensional frequency-domain received signal vector of the $m$ th OFDM symbol over frequency selective fading channels can be expressed as

$$
\boldsymbol{R}_{m}=\boldsymbol{G}_{m} \boldsymbol{H}_{m} \boldsymbol{x}_{m}+\boldsymbol{W}_{m}
$$

where $\boldsymbol{x}_{m}$ is the $N$-dimensional transmitted symbol vector with $\left[\boldsymbol{x}_{m}\right]_{k}=X_{m}(k), \boldsymbol{W}_{m}$ is an $N$-dimensional AWGN vector with the covariance matrix $\sigma_{W}^{2} \boldsymbol{I}_{N}$ and $\boldsymbol{H}_{m}$ is the frequency-domain channel matrix defined as $\boldsymbol{H}_{m}=$ $\operatorname{diag}\left(H_{m}(0), \cdots, H_{m}(N-1)\right) . \quad$ In $(1)$, the $N$-by- $N$ matrix $\boldsymbol{G}_{m}$ is the frequency-domain PHN matrix given as $\boldsymbol{G}_{m}=\boldsymbol{F}^{H} \varphi_{m} \boldsymbol{F}$ where $\boldsymbol{F}$ is the $N$-point IDFT matrix with $[\boldsymbol{F}]_{n, k}=(1 / \sqrt{N}) e^{j(2 \pi / N) n k}$ and PHN $\phi_{m}(n)$ is represented by the time-domain PHN matrix $\varphi_{m}$ given as $\boldsymbol{\varphi}_{m}=$ $\operatorname{diag}\left(e^{j \varphi_{m}(0)}, \cdots, e^{j \varphi_{m}(N-1)}\right)$. Here, $\boldsymbol{G}_{m}$ is split in $N$ matrices as follows:

$$
\boldsymbol{G}_{m}=g_{m, 0} \mathcal{I}_{0}+g_{m, 1} \mathcal{I}_{1}+\cdots+g_{m, N-1} \mathcal{I}_{N-1}
$$

where $g_{m, k}=(1 / N) \sum_{n=0}^{N-1} e^{-j(2 \pi / N) n k+j \varphi_{m}(n)}$ and $\mathcal{I}_{k}$ is an $N$ by- $N$ matrix given by $\left[\mathcal{I}_{k}\right]_{p, q}=1$ if $((q-p))_{N}$ equals to $k$ and $\left[\mathcal{I}_{k}\right]_{p, q}=0$ otherwise. It is seen that $g_{m, 0}$ denotes the common phase error (CPE) and $\sum_{k=1}^{N-1} g_{m, k} \mathcal{I}_{k} \boldsymbol{H}_{m} \boldsymbol{x}_{m}$ implies the ICI due to PHN. Moreover, $\phi_{m}(n)$ can be modeled as a discrete Wiener process by $\phi_{m}(n)=\sum_{i=0}^{m\left(N+N_{C P}\right)+N_{C P}+n} u(i)$ where $u(i)$ is a mutually independent zero mean Gaussian random variable [4]. The variance of $u(i)$ is given by $\sigma_{u}^{2}=$ $2 \pi \beta T_{s} / N$ where $T_{s}$ is the symbol period and $\beta$ denotes the two-sided 3-dB linewidth. The correlation function of $e^{j \phi_{m}(n)}$ is shown to be [4]

$$
E\left[e^{j \phi_{m}(p)} e^{-j \phi_{m}(q)}\right]=e^{-\pi \beta T_{s}|p-q| / N} .
$$

Assuming ideal timing synchronization and sufficient $\mathrm{CP}$, the channel frequency matrix $\boldsymbol{H}_{m}$ can be given as $\boldsymbol{H}_{m}=$ $\operatorname{diag}\left\{\boldsymbol{D} \boldsymbol{h}_{m}\right\}$ where $\boldsymbol{h}_{m}$ is a $L$-dimensional channel impulse response (CIR) vector and $\boldsymbol{D}$ is the $N$-by- $L$ DFT matrix with $[\boldsymbol{D}]_{k, l}=e^{-j(2 \pi / N) k l}$.

By defining a new effective CIR vector $\boldsymbol{\eta}_{m, k}=g_{m, k} \boldsymbol{h}_{m}$, 
(1) can be rewritten as

$$
\boldsymbol{R}_{m}=\mathcal{X}_{m} \mathcal{D} \boldsymbol{\eta}_{m}+\boldsymbol{W}_{m}
$$

where $\mathcal{X}_{m}=\left[\mathcal{X}_{m, 0} \cdots \mathcal{X}_{m, N-1}\right]$ with $\mathcal{X}_{m, k}=\mathcal{I}_{k} \operatorname{diag}\left\{\boldsymbol{x}_{m}\right\}, \mathcal{D}$ is a $N^{2}$-by- $N L$ matrix given by $\mathcal{D}=\operatorname{diag}(\boldsymbol{D}, \cdots, \boldsymbol{D})$ and $\boldsymbol{\eta}_{m}=\left[\boldsymbol{\eta}_{m, 0}^{T} \cdots \boldsymbol{\eta}_{m, N-1}^{T}\right]^{T}$. Assuming that the CIR vector $\boldsymbol{h}_{m}$ is a zero mean complex Gaussian random variable with the covariance matrix $\boldsymbol{C}_{h}$ [6], $\boldsymbol{\eta}_{m}$ can be modeled as a zero mean Gaussian vector with the covariance matrix $\boldsymbol{C}_{\eta}$. The $N L$ by- $N L$ matrix $C_{\eta}$ is partitioned into $L$-by- $L$ blocks and the $(p, q)$ th block is denoted by $\boldsymbol{C}_{\eta}(p, q)$. Since $\boldsymbol{G}_{m}$ and $\boldsymbol{\eta}_{m}$ are independent, $\boldsymbol{C}_{\eta}(p, q)$ is given by

$$
\boldsymbol{C}_{\eta}(p, q)=E\left[\boldsymbol{\eta}_{m, p} \boldsymbol{\eta}_{m, q}^{H}\right]=\sigma_{g}^{2}(p, q) \boldsymbol{C}_{h}
$$

where $\sigma_{g}^{2}(p, q)=E\left[g_{m, p} g_{m, q}^{*}\right]$ can be obtained by [3]

$$
\sigma_{g}^{2}(p, q)=\frac{1}{N^{2}} \sum_{n 1=0}^{N-1} \sum_{n 2=0}^{N-1} e^{-\pi \beta T_{s}|n 1-n 2| / N} e^{-j 2 \pi(n 1 p-n 2 q) / N} .
$$

\section{Joint Channel Estimation and Phase Noise Suppres- sion}

In the proposed PADD approaches, the initial effective CIRs are estimated using pilot sub-carriers, the tentative data symbol can be obtained using the estimates of effective CIRs and the effective CIRs are re-estimated in the decision-directed (DD) manner. Even when the DD approach is used, we have available only $N$ values for the effective CIRs of $N L$ parameters. To reduce the number of parameters needed for the effective CIRs estimation, the effective CIRs which significantly affect $\boldsymbol{R}_{m}$ in (4) are used.

The frequency-domain received signal vector at the pilot sub-carrier set $S_{P}=\left\{p(0), \cdots, p\left(N_{P}-1\right)\right\}$ can be given by

$$
\boldsymbol{R}_{m}^{P}=\left(\sum_{k=0}^{N-1} g_{m, k} \mathcal{I}_{k}^{P}\right) \boldsymbol{H}_{m}^{P} \boldsymbol{x}_{m}^{P}+\boldsymbol{V}_{m}^{P}+\boldsymbol{W}_{m}^{P}
$$

where the $N_{P}$-by- $N_{P}$ matrix $\boldsymbol{H}_{m}^{P}$ is the channel frequency matrix at the pilot sub-carriers, $\boldsymbol{x}_{m}^{P}$ denotes the $N_{P}$-dimensional pilot symbol vector, $\boldsymbol{W}_{m}^{P}$ is the the $N_{P^{-}}$ dimensional AWGN at the pilot sub-carriers, $\boldsymbol{V}_{m}^{P}$ is the $N_{P^{-}}$ dimensional vector with $\left[\boldsymbol{V}_{m}^{P}\right]_{i}=\sum_{k=0}^{N-1} \sum_{q \in S_{D}} g_{m, k}\left[\mathcal{I}_{k}\right]_{p(i), q}$ $H_{m}(q) X_{m}(q)$ and $\mathcal{I}_{k}^{P}$ is an $N_{P}$-by- $N_{P}$ matrix given by $\left[\mathcal{I}_{k}^{P}\right]_{i, j}=1$ if $((p(j)-p(i)))_{N}$ equals to $k$ and $\left[\mathcal{I}_{k}^{P}\right]_{i, j}=0$ otherwise.

Since most energy of the frequency responses of PHN is concentrated in the neighborhood of the CPE $g_{m, 0}$ [3], we try to estimate the initial effective CIR vector $\boldsymbol{\eta}_{m, \mathcal{P}(i)}=$ $g_{m, \mathcal{P}(i)} \boldsymbol{h}_{m}$ with the index set $\mathbb{P}=\{\mathcal{P}(0), \cdots, \mathcal{P}(2 P)\}$ where $\mathbb{P}$ $=\{\mathcal{P}(i) ; 0 \leq \mathcal{P}(i) \leq P$ or $N-P \leq \mathcal{P}(i)<N\}$ and $P$ is the order of the estimation for the initial effective CIRs. For example, we estimate $\boldsymbol{\eta}_{m, 0}=g_{m, 0} \boldsymbol{h}_{m}$ using pilot symbols when $P=0$. When $P=1$, we estimate $\boldsymbol{\eta}_{m, 0}=g_{m, 0} \boldsymbol{h}_{m}, \boldsymbol{\eta}_{m, 1}=$ $g_{m, 1} \boldsymbol{h}_{m}$ and $\boldsymbol{\eta}_{m, N-1}=g_{m, N-1} \boldsymbol{h}_{m}$ using pilot symbols.

Considering the $(2 P+1) L$-dimensional vector $\boldsymbol{\eta}_{m}^{P}=$ $\left[\boldsymbol{\eta}_{m, \mathcal{P}(0)}^{T} \cdots \boldsymbol{\eta}_{m, \mathcal{P}(2 P)}^{T}\right]^{T},(7)$ can be expressed as

$$
\boldsymbol{R}_{m}^{P}=\mathcal{X}_{m}^{P} \mathcal{D}^{P} \boldsymbol{\eta}_{m}^{P}+\mathcal{V}_{m}^{P}
$$

where $\mathcal{X}_{m}^{\mathcal{P}}=\left[\mathcal{X}_{m, \mathcal{P}(0)}^{P} \cdots \mathcal{X}_{m, \mathcal{P}(2 P)}^{P}\right]$ with $\mathcal{X}_{m, \mathcal{P}(i)}^{P}=\mathcal{I}_{\mathcal{P}(i)}^{P} \operatorname{diag}$

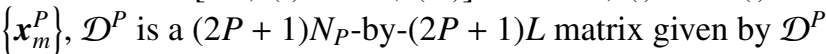
$=\operatorname{diag}\left(\boldsymbol{D}^{P}, \cdots, \boldsymbol{D}^{P}\right), \boldsymbol{D}^{P}$ is the DFT matrix of only pilot sub-carriers and $\mathcal{V}_{m}^{P}=\sum_{k \notin \mathbb{P}} g_{m, k} \mathcal{I}_{k}^{P} \boldsymbol{H}_{m}^{P} \boldsymbol{x}_{m}^{P}+\boldsymbol{V}_{m}^{P}+\boldsymbol{W}_{m}^{P}$.

Assuming independent and identically distributed (i.i.d.) data symbols, $\mathcal{V}_{m}^{P}$ can be approximated as a zeromean Gaussian vector of the covariance matrix of $\sigma_{\mathcal{V}^{P}}^{2} \boldsymbol{I}_{N_{P}}$ [2]. Using (8), the MLE for $\boldsymbol{\eta}_{m}^{P}$ can be obtained by [5]

$$
\hat{\boldsymbol{\eta}}_{m, M L E}^{P}=\left(\mathcal{B}_{m}^{P^{H}} \mathcal{B}_{m}^{P}\right)^{-1} \mathcal{B}_{m}^{P H} \boldsymbol{R}_{m}^{P}
$$

where $\mathcal{B}_{m}^{P}=\mathcal{X}_{m}^{P} \mathcal{D}^{P}$.

Assuming that $\boldsymbol{\eta}_{m}^{P}$ is uncorrelated with $\mathcal{V}_{m}^{P}$, the MMSEE can be given by [5]

$$
\hat{\boldsymbol{\eta}}_{m, M M S E E}^{P}=\left(\mathcal{B}_{m}^{P^{H}} \mathcal{B}_{m}^{P}+\sigma_{\mathcal{V}^{P}}^{2} \boldsymbol{C}_{\eta^{P}}^{-1}\right)^{-1} \mathcal{B}_{m}^{P^{H}} \boldsymbol{R}_{m}^{P}
$$

where $\boldsymbol{C}_{\eta^{P}}$ is the covariance matrix of $\boldsymbol{\eta}_{m}^{P}$. As shown in (10), the MMSEE needs $\boldsymbol{C}_{\eta^{P}}$ and $\sigma_{\mathcal{V}^{P}}^{2}$. In a practical system, the phase noise linewidth $\beta$ is known and the AWGN noise variance $\sigma_{W}^{2}$ can be determined from the receiver bandwidth and noise figure [4]. Then, $\boldsymbol{C}_{\eta^{P}}$ can be obtained using (5) and $\sigma_{\mathcal{V}^{P}}^{2}$ can be calculated as $\sigma_{\mathcal{V}^{P}}^{2}=\sum_{k \notin \mathbb{P}} \sigma_{g}^{2}(k, k)+\sigma_{W}^{2}$.

Using the estimate of effective CIRs $\hat{\boldsymbol{\eta}}_{m}^{P}=\left[\hat{\boldsymbol{\eta}}_{m, \mathcal{P}(0)}^{T} \cdots\right.$ $\left.\hat{\boldsymbol{\eta}}_{m, \mathcal{P}(2 P)}^{T}\right]^{T}$ from (9) or (10), we can rewrite (1) as

$$
\boldsymbol{R}_{m}=\mathcal{H}_{m} \boldsymbol{x}_{m}+\sum_{k=0, k \notin \mathbb{P}}^{N-1} g_{m, k} \mathcal{I}_{k} \boldsymbol{H}_{m} \boldsymbol{x}_{m}+\boldsymbol{W}_{m}
$$

where $\mathcal{H}_{m}=\sum_{k \in \mathbb{P}} \mathcal{H}_{m, k}$ with $\mathcal{H}_{m, k}=\mathcal{I}_{k} \operatorname{diag}\left\{\boldsymbol{D} \hat{\boldsymbol{\eta}}_{m, k}\right\}$. Then, we can obtain the tentative data using $\hat{\boldsymbol{x}}_{m}=\mathcal{H}_{m}^{-1} \boldsymbol{R}_{m}$. When $P=0, \mathcal{H}_{m}=\mathcal{H}_{m, 0}$ is a diagonal matrix so the tentative data can be easily obtained. However, when $P \neq 0$, the inversion of the $N$-by- $N$ matrix $\mathcal{H}_{m}$ requires a computational complexity of $O\left(N^{3}\right)$. To reduce the computational complexity, we estimate the data symbol vector $\tilde{\boldsymbol{x}}_{m}$ using $\tilde{\boldsymbol{x}}_{m}=\mathcal{H}_{m .0}^{-1} \boldsymbol{R}_{m}$ and the tentative data symbol vector $\hat{\boldsymbol{x}}_{m}$ can be obtained by

$$
\hat{\boldsymbol{x}}_{m}=\mathcal{H}_{m, 0}^{-1}\left(\boldsymbol{R}_{m}-\sum_{k \in \mathbb{P}, k \neq 0} \mathcal{H}_{m, k} \tilde{\boldsymbol{x}}_{m}\right) .
$$

After obtaining $\hat{\boldsymbol{x}}_{m}$, we try to estimate the effective CIR vector $\boldsymbol{\eta}_{m, \mathcal{M}(i)}=g_{m, \mathcal{M}(i)} \boldsymbol{h}_{m}$ with the index set $\mathbb{M}=$ $\{\mathcal{M}(0), \cdots, \mathcal{M}(2 M)\}$ where $\mathbb{M}=\{\mathcal{M}(i) ; 0 \leq \mathcal{M}(i) \leq M$ or $N-P \leq \mathcal{M}(i)<N\}$ and $M$ is the order of the decisiondirected estimation for the effective CIRs. Given $\hat{\boldsymbol{x}}_{m}$, which is assumed to be the same as the transmitted symbol vector $\boldsymbol{x}_{m}$, (1) can be expressed as

$$
\boldsymbol{R}_{m}=\bar{\chi}_{m} \overline{\mathcal{D}} \overline{\boldsymbol{\eta}}_{m}+\mathcal{V}_{m}
$$


where $\bar{X}_{m}=\left[\mathcal{X}_{m, \mathcal{M}(0)} \cdots \mathcal{X}_{m, \mathcal{M}(2 M)}\right], \overline{\mathcal{D}}$ is a $(2 M+1) N$-by$(2 M+1) L$ matrix given by $\overline{\mathcal{D}}=\operatorname{diag}(\boldsymbol{D}, \cdots, \boldsymbol{D}), \overline{\boldsymbol{\eta}}_{m}=$ $\left[\boldsymbol{\eta}_{m, \mathcal{M}(0)}^{T} \cdots \boldsymbol{\eta}_{m, \mathcal{M}(2 M)}^{T}\right]^{T}$ and $\mathcal{V}_{m}=\sum_{k \notin \mathbb{M}} \boldsymbol{g}_{m, k} \mathcal{I}_{k} \boldsymbol{H}_{m} \boldsymbol{x}_{m}+\boldsymbol{W}_{m}$ is the ICI-plus-noise vector. When $M=0$, we estimate $\boldsymbol{\eta}_{m, 0}$ $=g_{m, 0} \boldsymbol{h}_{m}$ using tentative data symbols. When $M=1$, we estimate $\boldsymbol{\eta}_{m, 0}=g_{m, 0} \boldsymbol{h}_{m}, \boldsymbol{\eta}_{m, 1}=g_{m, 1} \boldsymbol{h}_{m}$ and $\boldsymbol{\eta}_{m, N-1}=g_{m, N-1} \boldsymbol{h}_{m}$ using tentative data symbols. Similarly to $\mathcal{V}_{m}^{P}, \mathcal{V}_{m}$ can be approximated as a zero-mean Gaussian vector of the covariance matrix of $\sigma_{\mathcal{V}}^{2} \boldsymbol{I}_{N}=\left(\sum_{k \notin \mathbb{M}} \sigma_{g}^{2}(k, k)+\sigma_{W}^{2}\right) \boldsymbol{I}_{N}$ [2]. Using (13), the MLE for $\overline{\boldsymbol{\eta}}_{m}$ can be obtained by

$$
\hat{\boldsymbol{\eta}}_{m, M L E}=\left(\mathcal{B}_{m}^{H} \mathcal{B}_{m}\right)^{-1} \mathcal{B}_{m}^{H} \boldsymbol{R}_{m}
$$

where $\mathcal{B}_{m}=\bar{X}_{m} \overline{\mathcal{D}}$. Assuming that $\overline{\boldsymbol{\eta}}_{m}$ is uncorrelated with $\mathcal{V}_{m}$, the MMSEE can be given by

$$
\hat{\boldsymbol{\eta}}_{m, M M S E E}=\left(\mathcal{B}_{m}^{H} \mathcal{B}_{m}+\sigma_{\mathcal{V}}^{2} \boldsymbol{C}_{\bar{\eta}}^{-1}\right)^{-1} \mathcal{B}_{m}^{H} \boldsymbol{R}_{m}
$$

where $\boldsymbol{C}_{\bar{\eta}}$ is the covariance matrix of $\overline{\boldsymbol{\eta}}_{m}$. Using the estimate of effective CIRs $\hat{\boldsymbol{\eta}}_{m}=\left[\hat{\boldsymbol{\eta}}_{m, \mathcal{M}(0)}^{T} \cdots \hat{\boldsymbol{\eta}}_{m, \mathcal{M}(2 M)}^{T}\right]$ from (14) or (15), we estimate the final data symbol vector $\overline{\boldsymbol{x}}_{m}$ can be obtained by

$$
\overline{\boldsymbol{x}}_{m}=\hat{\mathcal{H}}_{m, 0}^{-1}\left(\boldsymbol{R}_{m}-\sum_{k \in \mathbb{M}, k \neq 0} \hat{\mathcal{H}}_{m, k} \hat{\overline{\boldsymbol{x}}}_{m}\right)
$$

where $\hat{\mathcal{H}}_{m, k}=\mathcal{I}_{k} \operatorname{diag}\left\{\boldsymbol{D} \hat{\boldsymbol{\eta}}_{m, k}\right\}$ and $\hat{\overline{\boldsymbol{x}}}_{m}=\hat{\mathcal{H}}_{m, 0}^{-1} \boldsymbol{R}_{m}$.

In frequency-selective fading channels without PHN, placing the pilot sub-carriers equispaced on the DFT grid is the optimal scheme [7]. However, to estimate the initial effective CIRs with $P \geq 1$ in the presence of PHN, pilot subcarriers should be grouped together. Moreover, pilot subcarriers are partitioned into equispaced groups for frequency selective fading channels. The impact of the placement of pilot sub-carriers is addressed in the following section.

\section{Simulation Results}

In our simulations, the DFT size $N$ is 256 , the number of data sub-carriers $N_{D}$ is 192 , the number of pilot sub-carriers $N_{P}$ is 64 and the CP length $N_{C P}$ is 32 . The total system bandwidth is $20 \mathrm{MHz}$ and the symbol period $T_{s}$ is $12.8 \mu \mathrm{s}$. We consider frequency-selective fading channels with $\boldsymbol{C}_{h}=$ $\operatorname{diag}\left(\sigma_{h_{0}}^{2}, \cdots, \sigma_{h_{L-1}}^{2}\right)$ and $\sigma_{h_{l}}^{2}=e^{-l / 4} / \sum_{l=0}^{L-1} e^{-l / 4}$. Here, we considered two delay spread conditions, 1) $L=4$ and 2) $L=8$. In $L=4$ delay condition, the mean excess delay and rms delay spread are $0.0596 \mu \mathrm{s}$ and $0.0545 \mu \mathrm{s}$, respectively [9]. In $L=8$ case, the mean excess delay is $0.1134 \mu$ s and rms delay spread is $0.104 \mu \mathrm{s}$. Each multipath is modeled as a zero mean complex Gaussian random variable so that it varies according to Rayleigh distribution in mobile environments [6]. It is assumed that the carrier frequency and vehicle speed are set to be $5 \mathrm{GHz}$ and $60 \mathrm{~km} / \mathrm{h}$, respectively. Moreover, the pilot sub-carriers are modulated by BPSK, the data sub-carriers are modulated by 16 QAM and one packet is composed of 20 OFDM symbols.

Note that the CIR length $L$ should be known in order

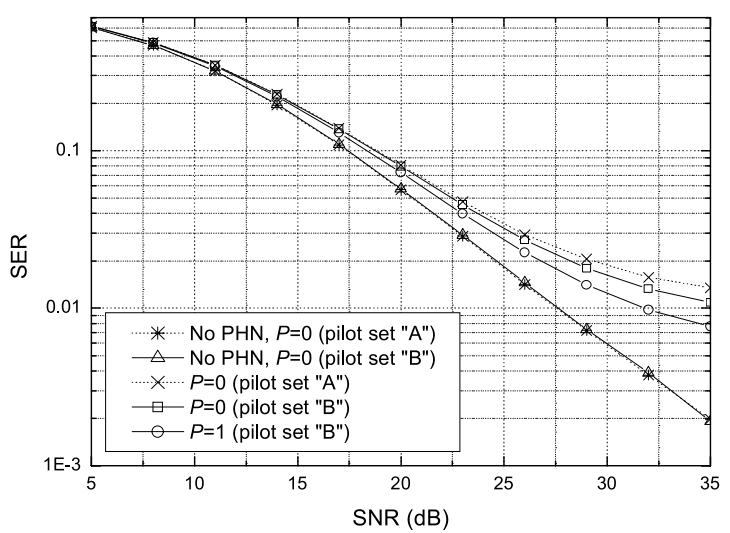

(a)

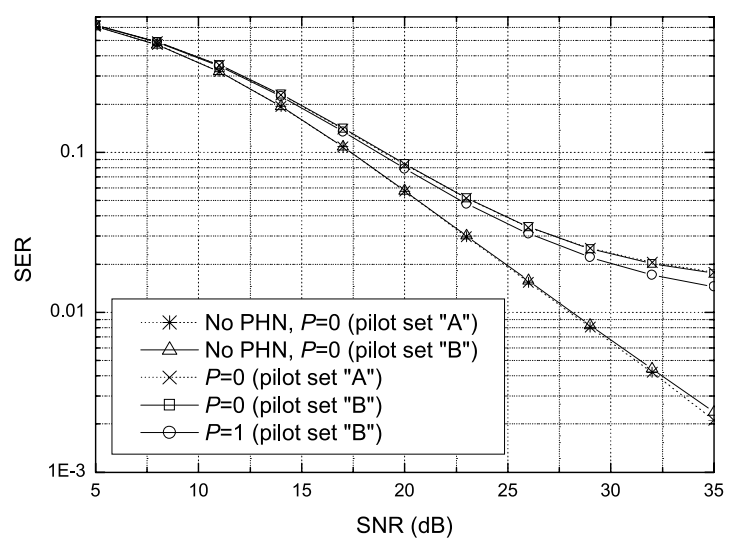

(b)

Fig. 1 SER performance of the MMSEE for the initial estimation with $\beta$ $=10^{3} \mathrm{~Hz}$. (a) $L=4$ (b) $L=8$.

to construct the MLE or the MMSEE. Here, we simply assume that the estimate of $L$ is equal to the CIR length [8]. Moreover, if $(2 P+1) L \leq N_{P}$ and $(2 M+1) L \leq N$, we can obtain the MLE or the MMSEE. In a practical system, $N$ is sufficiently larger than $3 L$. Therefore, when $3 L>N_{P}$, both the MLE and the MMSEE with $P=0$ and $M=1$ can be used and have good SER performances compared to both the MLE and the MMSEE with $P=M=0$. The argument of these approaches is confirmed by our simulations which are not illustrated here due to the limit of space.

For the comparison purposes, we consider two pilot sets. The pilot sub-carriers in the pilot set " $\mathrm{A}$ " are placed equispaced on the DFT grid where the pilot set "A" denotes $S_{P}=\left\{4 k+1 ; 0 \leq k<N_{P}\right\}$. The pilot sub-carriers in the pilot set "B" are placed into 16 groups of 4 pilot sub-carriers where the pilot set "B" is defined by $S_{P}=\{16 k+i+6$; $0 \leq k<16,0 \leq i<4\}$.

Figure 1 shows the SER performance versus the SNR for the MMSEE in (1) with $\beta=10^{3}$. Here, the MMSEE with no PHN and $P=0$ is equivalent to the MMSEE in [5]. For the MMSEE with no PHN and $P=0$, the SER performances with the pilot set "A" and "B" are comparable. Since the ICI effects from $\boldsymbol{\eta}_{m, 1}=g_{m, 1} \boldsymbol{h}_{m}$ and $\boldsymbol{\eta}_{m, N-1}=g_{m, N-1} \boldsymbol{h}_{m}$ are deleted, the MMSEE with $P=1$ and pilot set "B" has a better performance compared to the MMSEE with $P=$ 


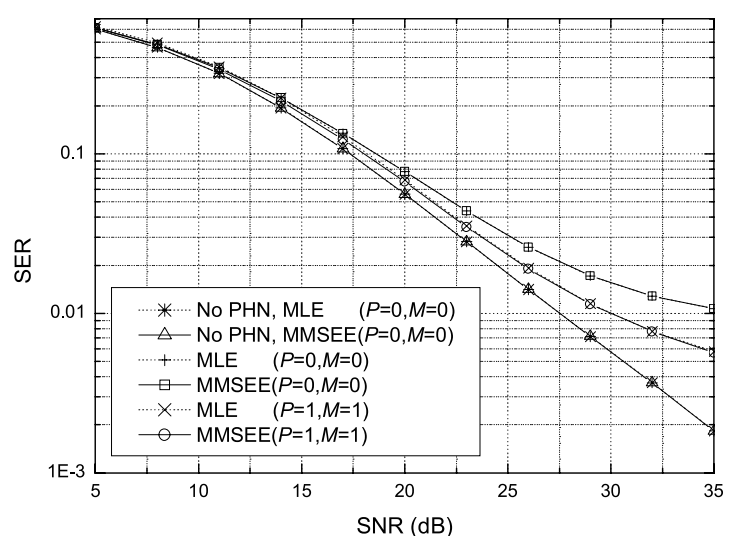

(a)

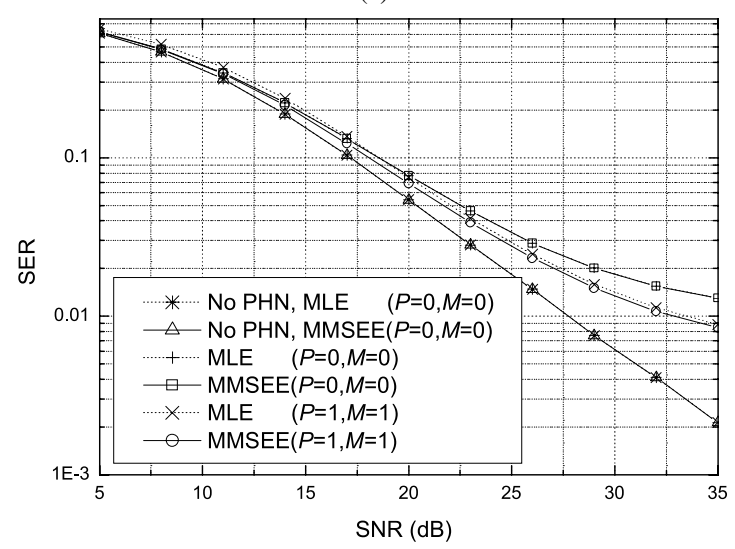

(b)

Fig. 2 SER performance versus SNR with the pilot set "B" and $\beta=$ $10^{3} \mathrm{~Hz}$. (a) $L=4$ (b) $L=8$.

0 and pilot set "B." In the proposed method, the larger the fading frequency selectivity is the smaller the channel correlation between sub-carriers and then the channel estimation error becomes large and the performance is degraded due to the low complexity decision directed estimation by Eq. (12). Therefore, as shown in Figs. 1(a) and (b), it is seen that the performance of the MMSEE with $P=1$, pilot set "B" and $L$ $=4$ is better than that of the MMSEE with $P=1$, pilot set "B" and $L=8$.

In Fig. 2, the SER performances for both the MLE and the MMSEE using the pilot set "B" are presented. When $P=M=0$, we estimate $\boldsymbol{\eta}_{m, 0}=g_{m, 0} \boldsymbol{h}_{m}$ using pilot symbols and tentative data symbols. In the case of the proposed schemes with $P=M=1, \boldsymbol{\eta}_{m, 0}=g_{m, 0} \boldsymbol{h}_{m}, \boldsymbol{\eta}_{m, 1}=g_{m, 1} \boldsymbol{h}_{m}$ and $\boldsymbol{\eta}_{m, N-1}=g_{m, N-1} \boldsymbol{h}_{m}$ are estimated using pilot symbols and tentative data symbols. Since the proposed schemes with $P=M=1$ eliminate the effects of $\boldsymbol{\eta}_{m, 1}=g_{m, 1} \boldsymbol{h}_{m}$ and $\boldsymbol{\eta}_{m, N-1}$ $=g_{m, N-1} \boldsymbol{h}_{m}$, the proposed schemes with $P=M=1$ outperform the MLE with $P=M=0$. When $P=M=0$, the SER performance for the MLE is almost the same as the SER performance for the MMSEE. For all the range of SNRs, when $P=M=1$, the MLE and the MMSEE have comparable performance.

Figure 3 shows the SER performance versus the SNR for the MMSEE with the pilot set "B" and $\beta=10^{3}$. When $P=M=2$ case, we estimate $\boldsymbol{\eta}_{m, 0}=g_{m, 0} \boldsymbol{h}_{m}, \boldsymbol{\eta}_{m, 1}=g_{m, 1} \boldsymbol{h}_{m}$,

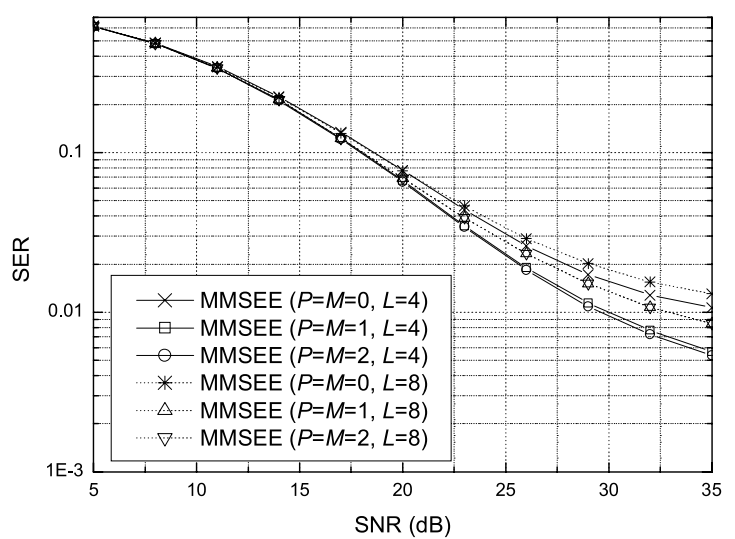

Fig. 3 SER performance versus SNR with the pilot set "B" and $\beta=$ $10^{3} \mathrm{~Hz}$.

$\boldsymbol{\eta}_{m, 2}=g_{m, 2} \boldsymbol{h}_{m}, \boldsymbol{\eta}_{m, N-1}=g_{m, N-1} \boldsymbol{h}_{m}$ and $\boldsymbol{\eta}_{m, N-2}=g_{m, N-2} \boldsymbol{h}_{m}$ using pilot symbols and tentative data symbols. In both $L$ $=4$ and $L=8$ cases, the MMSEE with $P=M=2$ has a negligible improvement compared to the MMSEE with $P=$ $M=1$. Considering the complexity of the algorithm, we select $P=1$ and $M=1$ for the proposed scheme.

\section{Conclusion}

In this letter, we propose joint channel estimation and PHN suppression schemes for OFDM systems based on the MLE and the MMSEE. It relies on a new parametric model that takes the CIR, the CPE and the ICI into account. With the obtained estimates, the low complexity detection method was proposed and the issue of pilot sub-carrier placement was examined. Simulation results show that the proposed schemes significantly compensate the performance degradation due to PHN.

\section{References}

[1] M. Speth, S.A. Fechtel, G. Fock, and H. Meyr, "Optimum receiver design for wireless broad-band systems using OFDM-part I," IEEE Trans. Commun., vol.47, no.11, pp.1668-1677, Nov. 1999.

[2] S. Wu and Y. Bar-Ness, "OFDM systems in the presence of phase noise: Consequences and solutions," IEEE Trans. Commun., vol.52, no.11, pp.1988-1996, Nov. 2004.

[3] D. Petrovic, W. Rave, and G. Fettweis, "Intercarrier interference due to phase noise in OFDM - Estimation and suppression," Proc. VTC04-Fall, 2004, pp.2191-2195.

[4] S. Wu, P. Liu, and Y. Bar-Ness, "Phase noise estimation and mitigation for OFDM systems," IEEE Trans. Wireless Commun., vol.5, no.12, pp.3616-3625, Dec. 2006.

[5] M. Morelli and U. Mengali, "A comparison of pilot-aided channel estimation methods for OFDM systems," IEEE Trans. Signal Process., vol.49, no.12, pp.3065-3073, Dec. 2001.

[6] W.C. Jakes, Microwave mobile communications, Wiley, New York, 1974.

[7] R. Negi and J. Cioffi, "Pilot tone selection for channel estimation in a mobile OFDM system," IEEE Trans. Consum. Electron., vol.44, no.3, pp.1122-1128, Aug. 1998.

[8] R. van Nee and R. Prasad, OFDM for Wireless Multimedia Communications, Artech House Publisher, 2000.

[9] T.S. Rappaport, Wireless communications: Principles \& Practice, Prentice Hall, NJ, 1996. 